Background and aims The PI3K pathway is frequently activated during tumourigenesis through deletion of the tumour suppressor PTEN. In contrast, increased PTEN expression in adipose tissue results in an increase in UCP1 expression and provides metabolic protection from tumourigenesis. This intrinsic protection normally arises from interscapular brown adipose tissue (iBAT) but may also arise from 'beiging' of inguinal white adipose tissue (iWAT). The aim of this study was to see if an association existed between UCP1 expression in adipose tissue and paediatric brain tumour growth through elevated PTEN levels.

Methods Two types of medulloblastoma (WNT and group 4) and ependymoma tumour cells were orthotopically xenografted into mice. iBAT and iWAT samples were extracted from tumour and non-tumour bearing mice to examine UCP1 and PTEN expression through QRT-PCR and Western blotting. Haematoxylin and eosin staining and UCP1 antibody immunohistochemistry (IHC) was also used to determine BAT activity in adipose tissue. Thermogenic activity of the adipose tissue was indirectly measured by thermal imaging of mice.

Results iWAT from ependymoma tumour-bearing mice had evidence of beiging and increased UCP1 expression through histology and IHC, while UCP1 expression in iBAT remained high in all mice. An increase in UCP1 gene expression and thermogenesis was observed with spinal metastasis. PTEN expression did not relate to UCP1 expression.

Conclusion Our data indicated mice implanted with aggressive tumours had increased UCP1 expression in iWAT. In conclusion, this pilot study suggests rapidly growing and metastatic brain tumours stimulate metabolic protection via an increase UCP1 expression in iWAT.

\section{Paediatric Emergency Medicine II}

\section{0-094 DIAGNOSTIC USEFULNESS OF BIOMARKERS IN THE MANAGEMENT OF CHILDREN WITH FEVER AT RISK OF SERIOUS BACTERIAL INFECTIONS AT THE EMERGENCY DEPARTMENT: PROSPECTIVE DIAGNOSTIC STUDY}

${ }^{1} \mathrm{RG}$ Nijman, ${ }^{2} Y$ Vergouwe, ${ }^{1} \mathrm{HA}$ Moll, ${ }^{3} \mathrm{WA}$ Dik, ${ }^{4} \mathrm{FJ}$ Smit, ${ }^{1} \mathrm{M}$ van Veen, ${ }^{5} \mathrm{~F}$ Weerkamp, ${ }^{2}$ EW Steyerberg, ${ }^{6}$ J van der Lei, ${ }^{7}$ YB de Rijke, ${ }^{1} \mathrm{R}$ Oostenbrink. ${ }^{1}$ Department of Pediatrics, Erasmus MC Sophia Children's Hospital, Rotterdam, Netherlands; ${ }^{2}$ Department of Public Health, Erasmus MC - University Medical Center Rotterdam, Rotterdam, Netherlands; ${ }^{3}$ Department of Immunology, Erasmus MC - University Medical Center Rotterdam, Rotterdam, Netherlands; ${ }^{4}$ Department of Pediatrics, Maasstad Hospital, Rotterdam, Netherlands; ${ }^{5}$ Department of Clinical Chemistry, Maasstad Hospital, Rotterdam, Netherlands; ${ }^{6}$ Department of Medical Informatics, Erasmus MC - University Medical Center Rotterdam, Rotterdam, Netherlands; ${ }^{7}$ Department of Clinical Chemistry, Erasmus MC - University Medical Center Rotterdam, Rotterdam, Netherlands

\subsection{6/archdischild-2014-307384.161}

Background and aims To evaluate the diagnostic usefulness of biomarkers in the management of children with fever at risk of serious bacterial infections (SBI) at the emergency department (ED).

Methods In this prospective observational study previously healthy children with fever, aged 1 month to 16 years, attending the EDs of a university hospital and a teaching hospital (Rotterdam, the Netherlands) between 2009 and 2012 were included. Standardised information on clinical signs and symptoms, Creactive protein (CRP), procalcitonin (PCT), neutrophil CD64 expression and urinalysis were collected prospectively. Logistic multivariable regression analysis was used to assess diagnostic performance.

Results 1,084 children were included, median age was 1.6 years (interquartile range: $0.8-3.5), 170$ children (16\%) had SBI. CRP (receiver operating characteristic curve (ROC-area) 0.77 (95\% confidence interval (CI) $0.69-0.85)$ ) and PCT (ROC-area 0.75 (95\% CI 0.67-0.83)) were both strong predictors of SBI. CD64 lacked diagnostic strength (ROC-area 0.62 (95\% CI 0.54-0.70)). A score containing PCT and CRP together with urinalysis, the Lab-score, performed well (ROC-area 0.79 (95\% CI $0.72-$ 0.87)), but thresholds performed similar to often used cut-offs of single biomarkers. Combined with clinical signs and symptoms both CRP and PCT were useful; additional PCT to CRP did not improve diagnostic performance substantially.

Conclusions CRP and PCT were equally useful in the diagnostic evaluation of the febrile child, whereas CD64 wasn't useful. Performing both CRP and PCT is often not indicated in a general population of febrile children. Our findings contrast previous studies suggesting PCT outperforming CRP and superior value of CD64 in specific settings.

\section{0-095 INFANTS WITH FRACTURES IN THE PAEDIATRIC EMERGENCY DEPARTMENT: ARE WE CONSIDERING CHILD PHYSICAL ABUSE?}

${ }^{1} \mathrm{~L}$ Lavin, ${ }^{2} \mathrm{C}$ Penrod, ${ }^{1} \mathrm{CM}$ Estrada, ${ }^{1} \mathrm{D}$ Arnold, ${ }^{3} \mathrm{X}$ Meng, ${ }^{3} \mathrm{~B}$ Saville, ${ }^{2} \mathrm{D}$ Lowen. ${ }^{1}$ Department of Pediatrics Division of Emergency Medicine, Vanderbilt University, Nashville, USA; ${ }^{2}$ Department of Pediatrics, Vanderbilt University, Nashville, USA; ${ }^{3}$ Department of Biostatistics, Vanderbilt University, Nashville, USA

\subsection{6/archdischild-2014-307384.162}

Background Recognition of child physical abuse (PA) is important to avoid further morbidity and mortality. There is limited knowledge regarding how frequently paediatric emergency department (PED) clinicians consider child abuse in infants with fractures.

Objective Estimate the percentage of infants with fractures for whom PA was considered, and to examine characteristics that are associated with consideration of PA.

Methods We reviewed the electronic medical record (EMR) of all patients $\leq 1$ year with fractures in a PED between 20082012. We used a multivariable logistic regression model to examine associations of patient and physician characteristics with our

\begin{tabular}{|c|c|}
\hline \multicolumn{2}{|l|}{ Patient characteristics } \\
\hline Age, in weeks ${ }^{a}$ & $27[12.1,38.7]$ \\
\hline Gender, Male ${ }^{\mathrm{b}}$ & $282(53)$ \\
\hline \multicolumn{2}{|l|}{ Race $^{b}$} \\
\hline Caucasian & $372(75)$ \\
\hline African American & $92(19)$ \\
\hline Hispanic & $21(4)$ \\
\hline \multicolumn{2}{|l|}{ Physician characteristics } \\
\hline Male $^{\mathrm{b}}$ & $282(53)$ \\
\hline PEM fellowship trained ${ }^{b, c}$ & $308(58)^{c}$ \\
\hline \multicolumn{2}{|l|}{ a. Median [IQR] } \\
\hline \multicolumn{2}{|l|}{ b. $n(\%)$} \\
\hline c. All others EM-trained except 1 paediatrics-trained & \\
\hline
\end{tabular}

